\title{
CONVERGENCE AND DIVERGENCE ALMOST EVERYWHERE OF SPHERICAL MEANS FOR RADIAL FUNCTIONS
}

\author{
YUICHI KANJIN
}

(Communicated by Richard R. Goldberg)

\begin{abstract}
Let $d>1$. It will be shown that the maximal operator $S^{*}$ of spherical means $S_{R}, R>0$, is bounded on $L^{p}\left(\mathbf{R}^{d}\right)$ radial functions when $2 d /(d+1)<p<2 d /(d-1)$, and it implies that, for every $L^{p}\left(\mathbf{R}^{d}\right)$ radial function $f(t), S_{R} f(t)$ converges to $f(t)$ for a.e. $t \in \mathbf{R}^{d}$ when $2 d /(d+1)<p \leq 2$. Also, it will be proved that there is an $L^{2 d /(d+1)}\left(R^{d}\right)$ radial function $f(t)$ with compact support such that $S_{R} f(t)$ diverges for a.e. $t \in R^{d}$.
\end{abstract}

Introduction. Define linear operators $S_{R}, R>0$, and $S^{*}$ on $L^{2}\left(R^{d}\right)$ by

$$
S_{R} f(t)=(2 \pi)^{-d / 2} \int_{|\xi| \leq R} \hat{f}(\xi) e^{i t \xi} d \xi
$$

and

$$
S^{*} f(t)=\sup _{R>0}\left|S_{R} f(t)\right|, \quad t \in \mathbf{R}^{d}
$$

respectively, where $\hat{f}(\xi)$ is the Fourier transform of a function $f(t)$, that is, $\hat{f}(\xi)=$ $(2 \pi)^{-d / 2} \int_{\mathbf{R}^{d}} f(t) e^{-i \xi t} d t, \xi \in \mathbf{R}^{d}$.

We shall assume for the rest of the paper that $d>1$. We put $S=S_{1}$. Fefferman [F] showed that $S$ is bounded on $L^{p}\left(\mathbf{R}^{d}\right)$ if and only if $p=2$. On the other hand, Herz $[\mathbf{H}]$ showed that, when restricted to $L^{p}\left(\mathbf{R}^{d}\right)$ radial functions, $S$ is bounded if and only if $2 d /(d+1)<p<2 d /(d-1)$. In this connection, Kenig and Tomas [KT1] showed that, for $p=2 d /(d+1)$ and $p=2 d /(d-1), S$ is not weak type on $L^{p}\left(\mathbf{R}^{d}\right)$ radial functions, and Chanillo $[\mathbf{C}]$ showed that $S$ is restricted weak type on $L^{p}\left(\mathbf{R}^{d}\right)$ radial functions for $p=2 d /(d+1)$.

This paper deals with boundedness of $S^{*}$ on $L^{p}\left(\mathbf{R}^{d}\right)$ radial functions. We shall prove a theorem which transplants a norm inequality for the maximal operators defined by Fourier Jacobi multipliers to a corresponding inequality for the maximal operators defined by Fourier Hankel multipliers. It follows from the theorem that $S^{*}$ is bounded on $L^{p}\left(\mathbf{R}^{d}\right)$ radial functions if $2 d /(d+1)<p<2 d /(d-1)$, which implies that $S_{R} f(t)$ converges to $f(t)$ almost everywhere for every $L^{p}\left(\mathbf{R}^{d}\right)$ radial function $f(t)$ if $2 d /(d+1)<p \leq 2$. We shall also show that there is an $L^{2 d /(d+1)}\left(\mathbf{R}^{d}\right)$ radial function $f(t)$ with compact support such that $S_{R} f(t)$ diverges almost everywhere.

Received by the editors April 17, 1987.

1980 Mathematics Subject Classification (1985 Revision). Primary 42B15; Secondary 42C10.

Key words and phrases. Maximal operator of spherical means for radial functions, a.e. convergence, a.e. divergence, transplantation theorem, Hankel transform. 
1. Statements of results. Let $\alpha \geq-1 / 2$. For a function $g(x)$ on the interval $(0, \infty)$, the Hankel transform $\tau_{\alpha} g(y), y \geq 0$, of order $\alpha$ is given by

$$
\tau_{\alpha} g(y)=\int_{0}^{\infty} g(x) \frac{J_{\alpha}(y x)}{(y x)^{\alpha}} x^{2 \alpha+1} d x
$$

where $J_{\alpha}(x)$ is the Bessel function of the first kind of order $\alpha$. We define

$$
L_{\alpha}^{p}=\left\{g(x) \text { on }(0, \infty) ;\|g\|_{\alpha, p}=\left(\int_{0}^{\infty}|g(x)|^{p} x^{2 \alpha+1} d x\right)^{1 / p}<\infty\right\} .
$$

Then it is known that $\left\|\tau_{\alpha} g\right\|_{\alpha, q} \leq C_{\alpha, p}\|g\|_{\alpha, p}$ for $g(x)$ in $L_{\alpha}^{p}$ if $1 \leq p \leq 2$, where $1 / p+1 / q=1$ and $C_{\alpha, p}$ is a constant depending only on $\alpha$ and $p$ (cf. $[\mathbf{H}]$ ).

Let $\lambda(y) \in L_{\alpha}^{\infty}$. Define operators $\Lambda_{R}, R>0$, and $\Lambda^{*}$ on $L_{\alpha}^{2}$ by

$$
\tau_{\alpha}\left(\Lambda_{R} g\right)(y)=\lambda(y / R) \tau_{\alpha} g(y) \text { and } \Lambda^{*} g(x)=\sup _{R>0}\left|\Lambda_{R} g(x)\right|
$$

respectively.

Let $P_{n}^{(\alpha, \beta)}(u)$ denote the Jacobi polynomial of degree $n$ and order $(\alpha, \beta), \alpha, \beta>$ -1 defined by

$$
(1-u)^{\alpha}(1+u)^{\beta} P_{n}^{(\alpha, \beta)}(u)=\frac{(-1)^{n}}{2^{n} n !}\left(\frac{d}{d u}\right)^{n}\left\{(1-u)^{n+\alpha}(1+u)^{n+\beta}\right\} .
$$

The functions $P_{n}^{(\alpha, \beta)}(\cos \theta)$ are orthogonal on $(0, \pi)$ with respect to

$$
d \mu(\theta)=(\sin \theta / 2)^{2 \alpha+1}(\cos \theta / 2)^{2 \beta+1} d \theta .
$$

We define

$$
\begin{aligned}
L_{(\alpha, \beta)}^{p}= & \{h(\theta) \text { on }(0, \pi) ; \\
& \left.\|h\|_{(\alpha, \beta), p}=\left(\int_{0}^{\pi}|h(\theta)|^{p} d \mu(\theta)\right)^{1 / p}<\infty\right\} .
\end{aligned}
$$

For a function $h(\theta)$ in $L_{(\alpha, \beta)}^{1}$, we have the Fourier Jacobi series

$$
h(\theta)=\sum_{n=0}^{\infty} \tilde{h}(n) \rho_{n} P_{n}^{(\alpha, \beta)}(\cos \theta)
$$

where

$$
\tilde{h}(n)=\int_{0}^{\pi} h(\theta) P_{n}^{(\alpha, \beta)}(\cos \theta) d \mu(\theta)
$$

and

$$
\rho_{n}=\left[\int_{0}^{\pi}\left\{P_{n}^{(\alpha, \beta)}(\cos \theta)\right\}^{2} d \mu(\theta)\right]^{-1} .
$$

Define operators $\tilde{\Lambda}_{R}, R>0$, and $\tilde{\Lambda}^{*}$ on $L_{(\alpha, \beta)}^{2}$ by

$$
\left(\tilde{\Lambda}_{R} h \tilde{)}(n)=\lambda(n / R) \tilde{h}(n) \quad \text { and } \quad \tilde{\Lambda}^{*} h(\theta)=\sup _{R>0}\left|\tilde{\Lambda}_{R} h(\theta)\right|,\right.
$$

respectively.

We are now ready to state the first theorem. 
THEOREM 1. Let $\alpha, \beta \geq-1 / 2$. Let $1<p<\infty$ and let $\lambda(y)$ be a function in $L_{\alpha}^{\infty}$ continuous for almost every $y$. If $\tilde{\Lambda}^{*}$ is bounded on $L_{(\alpha, \beta)}^{p}$, then $\Lambda^{*}$ is bounded on $L_{\alpha}^{p}$.

Define linear operators $T_{R}, R>0$, and $T^{*}$ on $L_{\alpha}^{2}$ by

and

$$
T_{R} g(x)=\int_{0}^{R} \tau_{\alpha} g(y) \frac{J_{\alpha}(x y)}{(x y)^{\alpha}} y^{2 \alpha+1} d y
$$

$$
T^{*} g(x)=\sup _{R>0}\left|T_{R} g(x)\right|, \quad x \geq 0
$$

respectively. Let $\chi_{(0,1)}(y)$ be the characteristic function of the interval $(0,1)$. For $\lambda(y)=\chi_{(0,1)}(y)$, we notice that $\Lambda^{*}=T^{*}$ and $\tilde{\Lambda}^{*}$ is the maximal operator of partial sum operators of the Fourier Jacobi series. From Badkov's theorem [B, Theorem 1.7], it follows that $\tilde{\Lambda}^{*}$ is bounded on $L_{(\alpha, \beta)}^{p}$ if

$$
\begin{aligned}
\max \{4(\alpha+1) /(2 \alpha+3), 4(\beta+1) /(2 \beta+3)\}<p \\
<\min \{4(\alpha+1) /(2 \alpha+1), 4(\beta+1) /(2 \beta+1)\}
\end{aligned}
$$

and $\alpha, \beta \geq-1 / 2$. Thus, Theorem 1 yields Corollary 1 from which Corollary 2 is obtained by using routine method.

COROLlaRY 1. Let $\alpha \geq-1 / 2$ and $4(\alpha+1) /(2 \alpha+3)<p<4(\alpha+1) /(2 \alpha+1)$. Then, $\left\|T^{*} g\right\|_{\alpha, p} \leq C\|g\|_{\alpha, p}$ for $g(x)$ in $L_{\alpha}^{p}$, where $C$ is a constant depending only on $\alpha$ and $p$.

COROLlary 2. Let $\alpha \geq-1 / 2$ and $4(\alpha+1) /(2 \alpha+3)<p \leq 2$. Then, for $g(x)$ in $L_{\alpha}^{p}, T_{R} g(x)$ converges to $g(x)$ for almost every $x$ in $(0, \infty)$ as $R \rightarrow \infty$.

By familiar relationship between Fourier transforms of radial functions and Hankel transforms, these corollaries are restated as follows.

CoRollary 3. Let $2 d /(d+1)<p<2 d /(d-1)$. Then, $\left\|S^{*} f\right\|_{p} \leq C\|f\|_{p}$ for $L^{p}\left(\mathbf{R}^{d}\right)$ radial functions $f(t)$, where $C$ is a constant depending only on $d$ and $p$.

COROLlaRY 4. Let $2 d /(d+1)<p \leq 2$. Then, for every $L^{p}\left(\mathbf{R}^{d}\right)$ radial function $f(t), S_{R} f(t)$ converges to $f(t)$ for almost every $t$ in $\mathbf{R}^{d}$ as $R \rightarrow \infty$.

On the divergence, the following theorem holds.

THEOREM 2. Let $\alpha>-1 / 2$. If $p=4(\alpha+1) /(2 \alpha+3)$, then there is a function $g(x)$ in $L_{\alpha}^{p}$ with compact support such that $T_{R} g(x)$ diverges almost everywhere in $(0, \infty)$ as $R \rightarrow \infty$.

COROLlaRY 5. If $p=2 d /(d+1)$, then there is an $L^{p}\left(\mathbf{R}^{d}\right)$ radial function $f(t)$ with compact support such that $S_{R} f(t)$ diverges almost everywhere in $\mathbf{R}^{d}$ as $R \rightarrow \infty$.

2. Proofs. A formulation (Lemma 1) of linearization of Kenig and Tomas [KT2] in our situation will enable us to prove Theorem 1 by following the proof of the theorem of Igari $[\mathbf{I}]$ which transplants a norm inequality for Fourier Jacobi multipliers to a corresponding inequality for Fourier Hankel multipliers. Theorem 2 will be proved by an argument similar to that used in Stanton and Tomas [ST] to prove divergence of central Fourier series on compact Lie groups, or in Meaney [M] to prove divergence of Jacobi polynomial series. See also Sogge [S]. 
LeMmA 1 [KT2, §1, LeMmA]. Let $1<p<\infty$ and $1 / p+1 / q=1$. Let $\lambda(y) \in L_{\alpha}^{\infty}$.

(i) The operator $\tilde{\Lambda}^{*}$ is bounded on $L_{(\alpha, \beta)}^{p}$ if and only if

$$
\left\|\sum_{k=1}^{\infty} \tilde{\Lambda}_{R_{k}} h_{k}\right\|_{(\alpha, \beta), q} \leq C\left\|\sum_{k=1}^{\infty}\left|h_{k}\right|\right\|_{(\alpha, \beta), q},
$$

where $C$ is a constant not depending on sequences $\left\{h_{k}(\theta)\right\}_{k=1}^{\infty} \subset L_{(\alpha, \beta)}^{q}$ and $\left\{R_{k}\right\}_{k=1}^{\infty}$, $R_{k}>0$.

(ii) The operator $\Lambda^{*}$ is bounded on $L_{\alpha}^{p}$ if and only if

$$
\left\|\sum_{k=1}^{\infty} \Lambda_{R_{k}} g_{k}\right\|_{\alpha, q} \leq C^{\prime}\left\|\sum_{k=1}^{\infty}\left|g_{k}\right|\right\|_{\alpha, q}
$$

where $C^{\prime}$ is a constant not depending on sequences $\left\{g_{k}(x)\right\}_{k=1}^{\infty} \subset L_{\alpha}^{q}$ and $\left\{R_{k}\right\}_{k=1}^{\infty}$, $R_{k}>0$.

Now we prove Theorem 1. Assume that (1) is valid. It suffices to show that (2) is valid for finite sequences $\left\{g_{k}(x)\right\}_{k=1}^{m}$ such that every $g_{k}(x)$ is an infinitely differentiable function with compact support in $(0, \infty)$. For $j>0$, define $g_{k, j}(\theta)=$ $g_{k}(j \theta)$. Then $\operatorname{supp} g_{k, j}(\theta) \subset(0, \pi)$ for large $j>0$ and $k=1,2,3, \ldots, m$. We consider $g_{k, j}(\theta)$ as a function belonging to $L_{(\alpha, \beta)}^{q}$. By the assumption, we have

$$
\left\|\sum_{k \in S} \tilde{\Lambda}_{j R_{k}} g_{k, j}\right\|_{(\alpha, \beta), q} \leq C\left\|\sum_{k \in S}\left|g_{k, j}\right|\right\|_{(\alpha, \beta), q},
$$

where $S$ is any subset of $\{1,2,3, \ldots, m\}$. Multiplying $\left\{j(2 j)^{2 \alpha+1}\right\}^{1 / q}$ by both sides of (3) and changing variable, we have

$$
\begin{aligned}
& \left\{\int_{0}^{j \pi}\left|\sum_{k \in S} G_{k}(x, j)\right|^{q} x^{2 \alpha+1}\left(\frac{2 j}{x} \sin \frac{x}{2 j}\right)^{2 \alpha+1}\left(\cos \frac{x}{2 j}\right)^{2 \beta+1} d x\right\}^{1 / q} \\
& \leq C\left\{\int_{0}^{j \pi}\left(\sum_{k \in S}\left|g_{k}(x)\right|\right)^{q} x^{2 \alpha+1}\left(\frac{2 j}{x} \sin \frac{x}{2 j}\right)^{2 \alpha+1}\left(\cos \frac{x}{2 j}\right)^{2 \beta+1} d x\right\}^{1 / q} \\
& \leq C\left\|\sum_{k \in S}\left|g_{k}\right|\right\|_{\alpha, q}
\end{aligned}
$$

where

$$
\begin{aligned}
G_{k}(x, j) & =\tilde{\Lambda}_{j R_{k}} g_{k, j}(x / j) \\
& =\sum_{n=0}^{\infty} \lambda\left(\frac{n}{j R_{k}}\right) \tilde{g}_{k, j}(n) \rho_{n} P_{n}^{(\alpha, \beta)}\left(\cos \frac{x}{j}\right) .
\end{aligned}
$$

Fix an arbitrary positive number $K$. For $j>3 K / 2 \pi$, we have

$$
\left(\frac{2 j}{x} \sin \frac{x}{2 j}\right)^{2 \alpha+1}\left(\cos \frac{x}{2 j}\right)^{2 \beta+1} \geq\left(\frac{\sqrt{3}}{2} / \frac{\pi}{3}\right)^{2 \alpha+1}\left(\frac{1}{2}\right)^{2 \beta+1}, \quad x \in(0, K) .
$$


Thus we have

$$
\left\{\int_{0}^{K}\left|\sum_{k \in S} G_{k}(x, j)\right|^{q} x^{2 \alpha+1} d x\right\}^{1 / q} \leq C^{\prime \prime}\left\|\sum_{k \in S}\left|g_{k}\right|\right\|_{\alpha, q}
$$

for $j>3 K / 2 \pi$, where $C^{\prime \prime}$ is a constant depending only on $\alpha, \beta$ and $q$. Notice that (4) is valid for $q=2$ since $\tilde{\Lambda}^{*}$ and $\Lambda^{*}$ are bounded on $L_{(\alpha, \beta)}^{2}$ and $L_{\alpha}^{2}$, respectively. Considering the case of $S=\{k\}, k=1,2,3, \ldots, m$, and taking a subsequence if necessary, we have that the sequence $\left\{G_{k}(x, j)\right\}_{j}$ converges weakly to a function $G_{k}(x)$ in $L_{\alpha}^{q}(0, K)$ and, simultaneously, in $L_{\alpha}^{2}(0, K)$ for every $k$, where $L_{\alpha}^{q}(0, K)=$ $\left\{g(x) \in L_{\alpha}^{q} ; \operatorname{supp} g(x) \subset(0, K)\right\}$. From the weak convergence of $\left\{G_{k}(x, j)\right\}_{j}$ in $L_{\alpha}^{q}(0, K)$ and the inequality (4) with $S=\{1,2,3, \ldots, m\}$, it follows that

$$
\left\{\int_{0}^{K}\left|\sum_{k=1}^{m} G_{k}(x)\right|^{q} x^{2 \alpha+1} d x\right\}^{1 / q} \leq C^{\prime \prime}\left\|\sum_{k=1}^{m}\left|g_{k}\right|\right\|_{\alpha, q} .
$$

To complete the proof, it suffices to obtain $G_{k}(x)=\Lambda_{R_{k}} g_{k}(x)$ a.e. $x \in(0, K)$ from the fact that $G_{k}(x)$ is the weak limit of $\left\{G_{k}(x, j)\right\}_{j}$ in $L_{\alpha}^{2}(0, K)$. But, it is a strict imitation of the method of [I, p. 203,l.10 ], and so we omit it.

Next we turn to the proof of Theorem 2. We divide the proof into two lemmas.

LEMMA 2. Let $\alpha>-1 / 2$ and $p=4(\alpha+1) /(2 \alpha+3)$. Define a sequence $\left\{\varphi_{k}\right\}_{k=1}^{\infty}$ of bounded linear functionals of the space $L_{\alpha}^{p}(0,1)$ by $\varphi_{k}(g)=\int_{k}^{k+1} \tau_{\alpha} g(y) y^{\alpha+1 / 2} d y$. Then the norms $\left\|\varphi_{k}\right\|$ of the functionals $\varphi_{k}$ satisfy that $\left\|\varphi_{k}\right\| \geq C(\log k)^{1 / q}$, where $q=4(\alpha+1) /(2 \alpha+1)$ and $C$ is a constant not depending on $k$.

LEMMA 3. Let $\alpha>-1 / 2$ and $(4 \alpha+2) /(2 \alpha+3) \leq p \leq 2$. If a function $g(x)$ in $L_{\alpha}^{p}$ satisfies the condition that $T_{R} g(x)$ converges on a set $E$ of positive measure, then $\lim _{R \rightarrow \infty} \int_{R}^{R+h} \tau_{\alpha} g(y) y^{\alpha+1 / 2} d y=0$ uniformly in $0 \leq h \leq 1$. that

By Lemma 2, there is a function $g(x)$ in $L_{\alpha}^{p}(0,1), p=4(\alpha+1) /(2 \alpha+3)$ such

$$
\lim \sup _{k \rightarrow \infty}\left|\int_{k}^{k+1} \tau_{\alpha} g(y) y^{\alpha+1 / 2} d y\right|=\infty
$$

It follows from Lemma 3 that $T_{R} g(x)$ diverges almost everywhere in $(0, \infty)$ as $R \rightarrow \infty$, which completes the proof of Theorem 2 .

PROOF OF LEMMA 2. By Fubini's theorem, we have

$$
\varphi_{k}(g)=\int_{0}^{1} g(x)\left\{\int_{k}^{k+1} \frac{J_{\alpha}(y x)}{(y x)^{\alpha}} y^{\alpha+1 / 2} d y\right\} x^{2 \alpha+1} d x
$$

and thus

$$
\left\|\varphi_{k}\right\|=\left(\int_{0}^{1}\left|\int_{k}^{k+1} \frac{J_{\alpha}(y x)}{(y x)^{\alpha}} y^{\alpha+1 / 2} d y\right|^{q} x^{2 \alpha+1} d x\right)^{1 / q}
$$

By the asymptotic formula

$$
J_{\alpha}(z)=\left(\frac{2}{\pi z}\right)^{1 / 2} \cos (z-\delta)+O\left(z^{-3 / 2}\right) \quad z \rightarrow \infty, \delta=(2 \alpha+1) \pi / 4
$$


we have

$$
\begin{aligned}
\left\|\varphi_{k}\right\| \geq & \left(\int_{1 / k}^{1}\left|\int_{k}^{k+1} \frac{J_{\alpha}(y x)}{(y x)^{\alpha}} y^{\alpha+1 / 2} d y\right|^{q} x^{2 \alpha+1} d x\right)^{1 / q} \\
\geq & C_{1}\left(\int_{1 / k}^{1}\left|\int_{k}^{k+1} \cos (y x-\delta) d y\right|^{q} x^{2 \alpha+1-q(\alpha+1 / 2)} d x\right)^{1 / q} \\
& -C_{2}\left(\int_{1 / k}^{1}\left|\int_{k}^{k+1} y^{-1} d y\right|^{q} x^{2 \alpha+1-q(\alpha+3 / 2)} d x\right)^{1 / q} \\
= & C_{1} A_{k}-C_{2} B_{k}, \text { say, }
\end{aligned}
$$

where $C_{1}$ and $C_{2}$ are positive constants not depending on $k$. The terms $B_{k}$ satisfy that

$$
B_{k}=\left\{\log \left(1+\frac{1}{k}\right)\right\}\left(\frac{k^{q}-1}{q}\right)^{1 / q}=O(1) \quad(k \rightarrow \infty) .
$$

By a simple calculation, we have

$$
\begin{aligned}
A_{k}^{q} & =2^{q} \int_{1 / k}^{1}\left|\cos \left(\left(k+\frac{1}{2}\right) x-\delta\right) \sin \frac{x}{2}\right|^{q} x^{-(q+1)} d x \\
& \geq\left(\frac{2}{\pi}\right)^{q} \int_{1 / k}^{1}\left|\cos \left(\left(k+\frac{1}{2}\right) x-\delta\right)\right|^{q} x^{-1} d x \\
& \geq C_{3} \log k
\end{aligned}
$$

where $C_{3}$ is a constant not depending on $k$. This completes the proof of Lemma 2 .

PROOF OF LEMMA 3. By Egorov's theorem, we have a closed set $E$ of positive measure such that $T_{R} g(x)$ converges uniformly on $E$. Then the integral

$$
U_{R}=\int_{R}^{R+h} \tau_{\alpha} g(y) \frac{J_{\alpha}(x y)}{(x y)^{\alpha}} y^{2 \alpha+1} d y
$$

converges to 0 as $R \rightarrow \infty$, uniformly in $x \in E$ and $0 \leq h \leq 1$. Without loss of generality, we may assume that $E \subset(a, b)$ with $0<a<b<\infty$ and $R \geq a^{-1}$. By the asymptotic formula (5), we have

$$
\begin{aligned}
U_{R}= & \frac{(2 / \pi)^{1 / 2}}{x^{\alpha+1 / 2}} \int_{R}^{R+h} \tau_{\alpha} g(y) \cos (x y-\delta) y^{\alpha+1 / 2} d y \\
& +\int_{R}^{R+h} \tau_{\alpha} g(y) O\left((x y)^{-3 / 2}\right)(x y)^{-\alpha} y^{2 \alpha+1} d y \\
= & \frac{(2 / \pi)^{1 / 2}}{x^{\alpha+1 / 2}} V_{R}+W_{R}, \quad \text { say, }
\end{aligned}
$$

and thus $\left|V_{R}\right| \leq C_{4}\left(\left|U_{R}\right|+\left|W_{R}\right|\right)$ for $x \in E$ and $0 \leq h \leq 1$, where $C_{4}$ is a constant depending only on $a, b$ and $\alpha$. We have

$$
\begin{aligned}
\left|W_{R}\right| & \leq C_{5}\left|\int_{R}^{R+h} \tau_{\alpha} g(y) y^{-(\alpha+3 / 2)} y^{2 \alpha+1} d y\right| \\
& \leq C_{5}\left(\int_{R}^{R+h}\left|\tau_{\alpha} g(y)\right|^{q} y^{2 \alpha+1} d y\right)^{1 / q} \cdot\left(\int_{R}^{R+h} y^{-p(\alpha+3 / 2)} y^{2 \alpha+1} d y\right)^{1 / p}
\end{aligned}
$$


where $C_{5}$ is a constant depending only on $a, b$ and $\alpha$. Since $\left\|\tau_{\alpha} g\right\|_{\alpha, q}<\infty$ and $(4 \alpha+2) /(2 \alpha+3) \leq p \leq 2$, it follows that $W_{R}=o(1)(R \rightarrow \infty)$ uniformly in $x \in E$ and $0 \leq h \leq 1$ which implies that $V_{R}=o(1)(R \rightarrow \infty)$ uniformly in $x \in E$ and $0 \leq h \leq 1$. We write $V_{R}$ in the form

$$
\int_{R}^{R+h} \cos x y d \chi_{1}(y)+\sin x y d \chi_{2}(y)
$$

where

$$
\begin{aligned}
& d \chi_{1}(y)=(\cos \delta) \tau_{\alpha} g(y) y^{\alpha+1 / 2} d y \\
& d \chi_{2}(y)=(\sin \delta) \tau_{\alpha} g(y) y^{\alpha+1 / 2} d y
\end{aligned}
$$

By the proof of $[\mathbf{Z}$, Chapter XVI Theorem (8.4)], which is a trigonometric integral analogue of the Cantor-Lebesgue theorem, we have that $\int_{R}^{R+h} d \chi_{j}(y)=o(1)(R \rightarrow$ $\infty)$ uniformly in $0 \leq h \leq 1$ for $j=1,2$ and thus

$$
\begin{aligned}
\int_{R}^{R+h} \tau_{\alpha} g(y) y^{\alpha+1 / 2} d y & =\int_{R}^{R+h} \cos \delta d \chi_{1}(y)+\sin \delta d \chi_{2}(y) \\
& =o(1) \quad(R \rightarrow \infty),
\end{aligned}
$$

uniformly in $0 \leq h \leq 1$. This completes the proof of Lemma 3 .

\section{REFERENCES}

[B] V. M. Badkov, Approximation properties of Fourier series in orthogonal polynomials, Russian Math. Surveys 33 (1978), 53-117.

[C] S. Chanillo, The multiplier for the ball and radial functions, J. Funct. Anal. 55 (1984), 18-24.

[F] C. Fefferman, The multiplier problem for the ball, Ann. of Math. (2) 94 (1971), 330-336.

[H] C. S. Herz, On the mean inversion of Fourier and Hankel transforms, Proc. Nat. Acad. Sci. U.S.A. 40 (1954), 996-999.

[I] S. Igari, On the multipliers of Hankel transform, Tôhoku Math. J. (2) 24 (1972), 201-206.

[KT1] C. E. Kenig and P. A. Tomas, The weak behavior of spherical means, Proc. Amer. Math. Soc. 78 (1980), 48-50.

[KT2] _ Maximal operators defined by Fourier multipliers, Studia Math. 68 (1980), 79-83.

[M] C. Meaney, Divergent Jacobi polynomial series, Proc. Amer. Math. Soc. 87 (1983), 459-462.

[S] C. Sogge, On the convergence of Riesz means on compact manifolds, Ann. of Math. (2) 126 (1987), 439-448.

[ST] R. J. Stanton and P. A. Tomas, Polyhedral summability of Fourier series on compact Lie groups, Amer. J. Math. 100 (1978), 477-493.

[Z] A. Zygmund, Trigonometric series, I and II, Cambridge Univ. Press, London, 1968.

Department of Mathematics, College of liberal Arts, Kanazawa UniverSITY, KANAZAWA 920, JAPAN 